\title{
La aproximación del desarrollo hacia un consenso global
}

\section{Pedro Caldentey del Pozo', José Juan Romero, S.J. ${ }^{2}$}

Resumen: El desarrollo está presente en la obra de los primeros economistas clásicos pero se consolida como disciplina específica a partir de la ll Guerra Mundial. Su evolución ha sido muy rica en amplitud y profundidad, pero en el periodo 1950-1990 se caracteriza por la presencia condicionante de dilemas o escuelas de pensamiento de naturaleza dicotómica (industria vs agricultura, neoliberalismo vs neoestructuralismo). En el periodo 1990-2020 se produce una cierta convergencia de las escuelas de pensamiento que permite la progresiva adopción de un consenso global sobre desarrollo hoy expresado en los Objetivos de Desarrollo Sostenible. La clave de esa convergencia son los conceptos de desarrollo humano y desarrollo sostenible. Estos dos conceptos han ofrecido un marco muy propicio para la convergencia y facilitado que las teorías del desarrollo adopten un enfoque multidimensional más cercano a la realidad y más sensible a los problemas de las más desfavorecidos y vulnerables.

Palabras clave: Desarrollo, desarrollo humano, desarrollo sostenible, crecimiento, desigualdad, ODS.

\footnotetext{
${ }^{1}$ Instituto Desarrollo Universidad Loyola Andalucía.

${ }^{2}$ Profesor Emérito, Universidad Loyola Andalucía.
} 


\section{Approaching a global consensus on development}

Abstract: The concept of development was already present in the works of the first classical economists though it only became a discipline in its own right afterWWII. Progress since then has been ample and profound. While the period between 1950 and 1990 was characterised by the conditioning presence of dilemmas or schools of thought of a dichotomous nature (industry vs agriculture, neoliberalism vs neostructuralism), a certain convergence took place between 1990 and 2020 that allowed for the adoption of a global consensus on development, which has found expression in the Sustainable Global Development Goals. The key to said convergence have been the concepts of human and sustainable development, which have offered a highly pertinent framework for this process and have allowed theories on development to adopt a multidimensional focus that more accurately reflects the realities and hardships experienced by the most vulnerable and underprivileged.

Key words: development, human development, sustainable development, growth, inequality, SDO.

\section{Vers un consensus mondiale sur le développement}

Résumé: Le développement est présent dans les travaux des premiers économistes classiques, mais s'est consolidé comme une discipline spécifique après la Seconde Guerre mondiale. Son évolution a été très riche en ampleur et en profondeur, mais dans la période 1950-1990, elle s'est caractérisée par la présence déterminante de dilemmes ou d'écoles de pensée de nature dichotomique (industrie contre agriculture, néo-libéralisme contre néo-structuralisme). Au cours de la période 1990-2020, il y a eu une certaine convergence des écoles de pensée qui a permis l'adoption progressive d'un consensus mondial sur le développement, aujourd'hui exprimé dans les Objectifs de Développement Durable. La clé de cette convergence réside dans les concepts de développement humain et de développement durable. Ces deux concepts ont fourni un cadre de convergence très favorable et ont facilitél'adoption par les théories du développement d'une approche multidimensionnelle, plus proche de la réalité et plus sensible aux problèmes des plus défavorisés et des plus vulnérables.

Mots clé: développement, développement humain, développement durable, croissance, inegalité, ODD.

\section{Introducción}

El desarrollo y su estudio ha sido un asuntos siempre presente en la teoría, pero nuevo como disciplina ya su vigencia contemporánea se puede establecer desde la II GM. Nace de un sistema multilateral de relaciones internacionales que, tras los horrores de la guerra, se basa en el compromiso con un principio de solidaridad y de responsabilidad compartida por el bienestar global y el respeto de los derechos humanos de cada persona allá donde se encuentre. 
Ya sea como disciplina o como asunto de interés general, el desarrollo es un tema complejo. Sus dimensiones son muy variadas y su estudio multidisciplinar. Las variables a las que se asocia forman una lista inagotable. El desarrollo nos empuja a estudiar el crecimiento del PIB, la pobreza y la inequidad, la transformación productiva, la agricultura y mundo rural, la política industrial, la innovación, demografía, educación, mercado laboral, salud, el sistema financiero, la actividad del sector público, el agotamiento de los recursos naturales o el cambio climático. Hemos aprendido que tenemos que hablar de libertades, gobernanza, cultura, de historia, de igualdad de género, del comportamiento de las personas y de las comunidades o de las relaciones de poder en todos sus ámbitos.

Pese a historia relativamente reciente, las primeras contribuciones de los clásicos antes del siglo XX estaban ya centrados en el desarrollo y lo hacían con una mirada global, aplicable a cualquier país del mundo. Hay un nexo evidente entre David Hume, John Stuart Mill, Karl Marx o La Riqueza de las Naciones de Adam Smith con los debates suscitados, por ejemplo, por ¿Por qué fracasan los países? (Acemoglu y Robinson 2012), una de las más relevantes entre las contribuciones recientes al desarrollo

La evolución del desarrollo y de la teoría y la práctica asociada a su naturaleza y evolución ha cambiado significativamente a lo largo de últimos setenta y cinco años. Lo ha hecho además para bien porque ha experimentado un tránsito desde el economicismo a la multidimensionalidad, porque se ha aproximado al interés común y al de los más desfavorecidos, porque ha ganado en complejidad y cercanía a la realidad, y porque se ha dotado de consensos globales que nos permiten superar falsos dilemas.

El desarrollo se enfrenta también a dificultades metodológicas como tantas otras disciplinas. Desde la debatida universalidad de las teorías y leyes del desarrollo cuando se aplican a contextos tan diversos, a la dificultad de establecer relaciones causales o líneales entre las variables. Todo parece indicar que la democracia, la libertad y el buen funcionamiento del estado o de los mercados tiene una alta correlación con el crecimiento y el bienestar. Pero qué va antes, como forzar un círculo virtuoso entre los factores determinantes del desarrollo (Yoh 2019).

En este inicio de siglo XXI ha sido recurrente la cuestión de si el mundo, el desarrollo, va a mejor o a peor. Hay dos factores que empujan al fatalismo para responder a esta pregunta. Por una parte, la propia complejidad del tema, su naturaleza multidisciplinaria y la confluencia de perspectivas no siempre fáciles de encajar, que han generado dificultades para entender los problemas o valorar la relevancia 
de los avances que se producen en torno a ellos. Por otra parte, la abundante información que nos inunda, nos aproxima a los problemas y nos hace más críticos y conscientes, aunque con cierto sesgo hacia lo cercano, lo inmediato y lo sensacionalista. Los dos factores nos hacen pensar en que todo tiende a empeorar.

Nadie ha combatido esa falsa percepción mejor que Hans Rosling, cuya plataforma gapminder (https://www.gapminder.org//) aporta rotundas evidencias con sus gráficos de bolas y sus videos divulgativos sobre nuestros errores de percepción y sobre cómo los problemas del desarrollo no paran de mejorar en todos los países del mundo. La polémica suscitada por trabajos como los de Steven Pinker resume bien el alcance de este debate. La percepción de estancamiento económico y de un creciente número de conflictos o violencia no se puede sostener con evidencias, pese a nuestra mayor conciencia de los problemas y a la presión de cierto populismo de carácter tribalista (Pinker 2018).

El mundo y las circunstancias que nos rodean no paran de mejorar y eso hace más difícil de aceptar el sufrimiento de los desfavorecidos o de los afectados por alguna de las formas de la violencia o los riesgos de futuro de una sociedad basada en un voraz uso del planeta. De ahí han surgido en la agenda del desarrollo una serie de temas emergentes que dominan hoy el análisis y la política. De entre ellos destacan con fuerza, por ejemplo, la desigualdad o el cambio climático.

\section{El pensamiento económico sobre desarrollo en el siglo $X X$ y la lucha de contrarios (1950-1990)}

La identificación del desarrollo con el crecimiento ha sido una constante en la teoría económica y durante buena parte de su evolución en el siglo XX. Desde la función del crecimiento, dependiente del factor trabajo, del factor capital y su productividad, las contribuciones de la teoría neoclásica fueron completando su análisis con la suma de determinantes exógenos (modelo Harrod-Domar) o con la idea del progreso técnico, o residuo de Solow, que está en la base de la hoy tan debatida productividad total de los factores. Después, los modelos de crecimiento endógeno añadieron a la ecuación los efectos del capital humano, de la innovación o del conocimiento para entender con mayor precisión la idea del progreso técnico y defendiendo su fundamento en factores endógenos o propios de cada economía.

El pensamiento y las teorías del desarrollo estuvieron marcadas durante toda la segunda mitad del siglo XXI por dilemas (industria vs agricultura, mercado vs es- 
tado) o juego de contrarios (ortodoxia vs heterodoxia, neoliberal vs neoestructural) que luego no han resultado muy útiles para entender el desarrollo y extenderlo a todas las regiones del mundo.

A partir de las contribuciones de la teoría neoclásica, tras los acuerdos de Breton Woods las políticas de desarrollo se inspiraron en el enfoque de las teorías estructuralistas, claramente más alejadas del Camino a la servidumbre de Hayek que de la Gran Transformación de Polanyi (Harris 2014) o de la Teoría General del empleo, el interés y el dinero de Keynes, dotando de un papel destacado al Estado y a la regulación de los mercados e incluso de las relaciones internacionales.

Las teorías ortodoxas del desarrollo tienen un antecedente temprano en las conocidas etapas del crecimiento de Rostow, que contribuyeron también en los años cincuenta a definir las bases conceptuales del salto al desarrollo a partir de la industrialización y los flujos de capital y trabajo entre sectores. Aunque como teoría quedo ampliamente superada, es fácil detectar como su planteamiento lineal y economicista y su tendencia a universalizar los procesos, sobreviven en la práctica del desarrollo y en sus prescriptores.

Desde finales de los años setenta, dadas las dificultades para asegurar un crecimiento sostenido en muchos países en desarrollo y con los efectos de las dos crisis de petróleo, el predominio de la propuesta neoestructuralista va dejando paso al enfoque neoliberal que ha proporcionado el marco de las políticas de desarrollo hasta la llegada de la Gran Recesión.

Las propuestas neoliberales se hacen predominantes a partir de los años ochenta. El llamado Consenso de Washington representa su mejor imagen a partir del decálogo en el que John Williamson recoge las condiciones implícitas o explícitas para recibir préstamos desde el $\mathrm{FMI}$, el BM o el tesoro estadounidense. Las condiciones se centraban en tres grandes principios: (a) políticas macroeconómicas sólidas (baja inflación, déficits públicos mínimos y equilibrios en las cuentas exteriores); (b) liberalización y apertura comercial y financiera y (c) promoción del papel del mercado, limitando el del Estado, a través de la privatización y la desregulación de los mercados.

Las ideas representadas por el Consenso de Washington desplazan en esta época los determinantes del crecimiento hacia los equilibrios de la economía neoclásica que propone recetas universales para el crecimiento, prestando así menor atención a los inputs o factores de producción que eran el objeto principal de la estrategia de crecimiento basada en industrialización. La propuesta neoliberal, largamente 
vigente, comparte con Rostow su sesgo hacia la linealidad y economicismo. Las críticas a su implementación abrirán paso a la concepción multidimensional del desarrollo.

Las primeras propuestas estructuralistas y la ortodoxia neoclásica convivieron durante la mitad del siglo XXI con las propuestas heterodoxas, inspiradas en las falencias de las teorías del crecimiento y el desarrollo. Estas propuestas, procedentes de la industrialización sustitutiva de importaciones de Raul Prebisch en la CEPAL, de la teoría de la dependencia (Andre Gunder Frank, Fernando Henrique Cardoso, Samir Amin) o del neomarxismo, planteaban que el desarrollo no era una fase de transición que se pudiera eliminar superando etapas o aplicando decisiones económicas, porque el sistema económico mundial se mantiene sobre estructuras que penalizan a los países en desarrollo y perpetúan las ventajas de los países más ricos (Consejo de Redacción RFS 2005, Harris 2014).

Este juego de contrarios o dialéctica teórica y política no impidió que fueran surgiendo planteamientos muy diversos y que se fuera gestando un contexto cada vez más difícil de encajar en las falsas dicotomías. La falta de resultados contundentes en el crecimiento de los países en desarrollo cuestionaba la validez de las teorías del desarrollo, tanto de las teorías de la modernización, como las de las estructuralistas. La crisis de la planificación socialista y del proyecto soviético también cuestionaba las propuestas derivadas de su modelo.

Por otra parte, algunos movimientos sociales de nuevo cuño ampliaron los términos del debate, entre ellos los movimientos los pacifistas, antinucleares o ecologistas ya presentes desde finales de los años setenta. El movimiento de los no alineados y el discurso anticolonialista que acompañó los procesos de independencia formaron parte de un entorno que cuestionaba cada vez más la teoría y práctica del desarrollo.

A finales de siglo, la descolonización, la caída del muro o la sensación de fracaso por no haber podido extender el desarrollo por todo el mundo, crearon las condiciones para abrir una etapa más centrada en los consensos que en los contrarios.

\section{La ruta hacia los consensos: el desarrollo humano y los ODS (1990-2020)}

Dos conceptos fundamentan las bases de un consenso global que va a modificar la dinámica y concepción del desarrollo en esta etapa. 
Por un parte, el concepto de desarrollo sostenible, con su definición en el Informe Nuestro futuro común o Informe Brundtland de la Comisión Mundial del medio ambiente y el desarrollo de Naciones Unidas: Aquel que satisface las necesidades del presente sin limitar el potencial para satisfacer las necesidades de las generaciones futuras. Un concepto que conecta la preocupación sobre el agotamiento de los recursos naturales y sobre el impacto de nuestra civilización en la tierra expresada en los primeros Informes sobre los Límites del Crecimiento del Club de Roma en 1972, con los informes del Grupo Intergubernamental de Expertos sobre el Cambio Climático (IPCC, por sus siglas en inglés) y con las Conferencias de las Partes (COP) que gestionan los acuerdos internacionales al respecto.

Por otra parte, el concepto de Desarrollo Humano que lanza el Programa de $\mathrm{Na-}$ ciones Unidas para el Desarrollo (PNUD) en 1990, junto a su popular Índice y que supone quizás la transformación más importante del debate sobre el desarrollo desde la creación de las Naciones Unidas. El concepto de desarrollo humano consolida muchas contribuciones anteriores a la teoría del desarrollo. Desde el concepto pionero del desarrollo multidimensional de Michael Todaro en su trabajo El desarrollo económico del Tercer Mundo de 1986 o los trabajos sobre pobreza en el entorno del Banco Mundial, hasta la reconocida influencia del pensamiento de Amartya Sen en la definición del desarrollo humano o de las aportaciones en torno al enfoque de capacidades humanas.

La relevancia del concepto de desarrollo humano se explica por tres razones. En primer lugar, porque es un concepto multidimensional, que abarca múltiples dimensiones del desarrollo, supera la idea de que el crecimiento es igual a desarrollo, y abandona el economicismo y la concepción lineal de las relaciones entre sus dimensiones. La dinámica de los círculos viciosos o virtuosos que se asocia al concepto y a sus informes, combate con rotundidad la simplicidad de la imagen del efecto catarata o Trickle-down que prometía el consenso de Washington ${ }^{3}$.

En segundo lugar, porque siguiendo a Amartya Sen sustituye la carencia de recursos por la carencia de opciones como base de su definición. El desarrollo se

\footnotetext{
${ }^{3}$ El efecto catarata o Trickle-Down ayudaba a explicar cómo la eficiente concentración de incentivos y recursos en los sectores de mayor renta, capacidad o conexión con los mercados internacionales, ayudaría a que esos sectores fueran capaces de generar mayor crecimiento y cómo éste se desbordaría en catarata a los sectores menos favorecidos a través del empleo y la inversión. En una frase que seguramente pervivirá en el tiempo, enunciada en medio del debate de la reducción de impuestos a los más ricos, el presidente Biden afirmó en una sesión conjunta del Congreso estadounidense que el efecto catarata nunca había funcionado (Trickle down has never worked)
} 
explica mejor en función de las capacidades, el objetivo básico del desarrollo es, por tanto, ampliar la capacidad de las personas (Consejo de redacción RFS 2005).

Es un avance significativo, en tercer lugar, porque centra el desarrollo en los que carecen de él o no lo alcanzan para vivir con dignidad. Como señalaba la célebre frase de Mahbuh Ul Haq, promotor del Informe y del índice (PNUD 2021): Nos han enseñado a ocuparnos del PNB para resolver el problema de la pobreza. Démosle la vuelta y ocupémonos de la pobreza para resolver el problema del PNB.

Aunque otros focos del debate sobre el desarrollo parecen haber robado la primera plana a los informes de desarrollo humano, las contribuciones que el PNUD ha ido realizando en todos ellos constituyen una trascedente aportación al análisis y al diseño de políticas. Se trata de un concepto, que, junto a su medición, no para de evolucionar. Por ejemplo, el informe del PNUD de 1992 definía así el desarrollo humano:

Proceso de ampliar la gama de opciones de las personas, brindándoles mayores oportunidades de educación, atención médica, ingreso y empleo, y abarcando el espectro total de opciones humanas, desde un entorno físico en buenas condiciones hasta libertades económicas y políticas (PNUD 1992).

En el informe del año 2010, que celebraba el vigésimo aniversario de su lanzamiento, lo definía así:

El desarrollo humano es la expansión de las libertades de las personas para llevar una vida prolongada, saludable y creativa; conseguir las metas que consideran valiosas y participar activamente en darle forma al desarrollo de manera equitativa y sostenible en un plantea compartido. Las personas son a la vez beneficiarias y agentes motivadores del desarrollo humano, como individuos y colectivamente (PNUD 2010).

En el informe más reciente, del año 2020, el PNUD introduce nuevos énfasis:

El desarrollo humano consiste en capacitar a las personas para que identifiquen y sigan su propio camino hacia una vida con sentido, basada en la ampliación de las libertades (PNUD 2020).

La discusión en torno a los conceptos de desarrollo humano y desarrollo sostenible y sus aportaciones al debate exceden las posibilidades de este artículo, aunque haremos alguna referencia posterior adicional. Los dos conceptos se divulgan y hacen universales en una sucesión de Cumbres en los primeros años noventa. Se trata de las Cumbres de Naciones Unidas sobre Medio Ambiente y Desarrollo (Rio de Janeiro 1992), sobre Población y Desarrollo (El Cairo 1994), la Cumbre sobre Desarrollo Social (Copenhague 1995) o la Cumbre sobre la Mujer (Beiijing 1995). Son reuniones sobre asuntos globales que se celebran con periodicidad 
cada década pero que en esos años tuvieron especial relevancia. La referencia a estas cumbres es común y frecuente porque actualizaron formalmente los conceptos y fueron adoptados globalmente para la negociación de acuerdos y la implementación de políticas.

No es aventurado afirmar que ambos conceptos y su adopción en las cumbres de los años noventa son el antecedente principal de los consensos globales que adoptamos en el siglo XXI en torno a los Objetivos del Milenio para el periodo 2000-2015 y los Objetivos de Desarrollo Sostenible para el periodo 2015-2030.

Hay una secuencia de contribuciones teóricas y acuerdos internacionales que enlaza la definición de los conceptos de desarrollo humano y sostenible, con las cumbres de los noventa y los ODS, pasando, por ejemplo, por los acuerdos sobre la eficacia de la ayuda del año 2005 o las Conferencias de Naciones Unidas sobre Cambio Climático. Esta secuencia domina el periodo 1990-2020 y se caracteriza primero porque, a diferencia del periodo 1950-1990, nos ha conducido al desarrollo de un sistema de consensos globales basado en adoptar lo mejor y más adecuado de las contribuciones de las distintas escuelas de pensamiento sobre desarrollo.

Los objetivos de desarrollo sostenible y los demás acuerdos globales sobre desarrollo descansan en una cierta convergencia (Hidalgo Capitán 1998) entre las diferentes teorías que acepta y combina la necesidad de evitar desequilibrios macroeconómicos que dificultan el crecimiento y el desempeño de los agentes económicos, con la necesidad de proteger el papel esencial que el estado debe realizar y con la necesidad de priorizar la atención a los más desfavorecidos con el objetivo prioritario de no dejar a nadie atrás.

Es también un consenso basado en la necesidad de encontrar el balance adecuado entre los roles del mercado y del estado, que reconoce la importancia de la multidimensionalidad del desarrollo, parece estar cómodo en el capitalismo liberal y los regímenes democráticos (Harris 2014) pero que demanda flexibilidad y diversidad institucional. El caso de Chima o los recientes brotes de autoritarismo que surgen en todas las regiones del mundo en desarrollo o desarrollado desafían esta percepción del consenso (Levintsky y Ziblatt 2018).

Se trata, en todo caso, de un escenario favorable y esperanzador. La evolución e interpretación de este consenso, así como la implementación de los ODS, está evidentemente sometido a riesgos e incertidumbres, pero construye una agenda universal que implica y obliga a todos los países. Se puede señalar que se trata de consensos muy amplios que no obligan a escoger. Pero los consensos estrechos y 
estrictos no habían funcionado tampoco, y no responden a la multidimensionalidad y naturaleza sistémica de nuestros problemas.

Es un proceso similar al que propone Dani Rodrik en torno a los cuestionamientos a la ciencia económica por su dificultad para adelantar la crisis, entre otras críticas. En su obra Las reglas de la economía, aciertos y errores de una ciencia en entredicho (Rodrik 2015), sin eludir el debate, defiende el valor de la economía, el uso de modelos matemáticos, pero, sobre todo, la importancia de entender que cada modelo y cada escuela de pensamiento es útil frente a determinados problemas y circunstancias, y renunciar a cualquiera de ellas desde posiciones dogmáticas parece poco inteligente. La reacción ante la crisis provocada por la pandemia y las restricciones de movilidad son un ejemplo de la capacidad de forjar consensos, de adaptarse a la especificidad de los problemas y de superar dogmas y dilemas innecesarios (Rodrik y Blanchard 2021).

\section{La medición del desarrollo}

La consolidación de los consensos globales en torno al desarrollo humano y sostenible y a la agenda 2030 está acompañada de otro proceso de gran interés para el desarrollo como es la necesidad de medir adecuadamente su evolución y características. Este avance tiene dos planos que no son exclusivos de la disciplina del desarrollo y sus debates. Por una parte, se trata de construir índices que respondan mejor a la complejidad de lo que se mide. Por otra parte, el rigor científico demanda cada vez más el respaldo de evidencias y datos, que no son siempre fáciles de conseguir.

La construcción de índices tiene una estrecha relación con el debate sobre las deficiencias del PIB para medir el desarrollo o el bienestar. Aunque el PIB no ofrece más de lo que promete, ha sido con frecuencia utilizado mucho más allá de sus posibilidades. Por eso fue tan importante el lanzamiento del Índice de Desarrollo Humano del PNUD y de sus posteriores índices complementarios, ajustados a la desigualdad o al género. Aunque el IDH no es capaz de abarcar todas las dimensiones del desarrollo humano, ofrece un indicador que supera la información proporcionada por el uso tradicional del PIB. En la comparación entre ambos surge de nuevo la ruptura entre la identificación del crecimiento con el desarrollo.

También en el ámbito de las economías más desarrolladas se trabaja en la elaboración de nuevos índices de desarrollo y bienestar. Un hito importante en este proceso fue el informe Medición del Desarrollo Económico y del Progreso Social, 
más conocido como Informe Stiglitz-Sen-Fitoussi (Stiglitz et al, 2009) e inspirado también en la comunicación de la Comisión Europea Más allá del PIB. Evaluación del progreso en un mundo cambiante publicada en agosto de 2009. A partir de estas iniciativas, los países de la UE han adoptado el Índice de Calidad de Vida que agrupa indicadores sobre nueve dimensiones que refuerzan la concepción multidimensional del bienestar (condiciones materiales de vida, trabajo, salud, educación, ocio y relaciones sociales, seguridad física y personal, gobernanza y derechos básicos, entorno y medioambiente, y bienestar subjetivo).

Existen además otros índices que han tenido cierta aceptación al menos como referencia. Entre los más destacados están el Índice para una Vida Mejor (Better Life Index) de la OCDE o el Índice de Progreso Social (Social Progress Index), junto a una larga lista animada por el interés de instituciones internacionales y del mundo académico por construir indicadores.

En el ámbito específico del desarrollo y la pobreza, es interesante destacar la adopción en la UE de la tasa AROPE (At Risk Of Poverty or Exclusion) que mide la pobreza o riesgo de pobreza a partir de tres dimensiones que van más allá de las tradicionales variables de renta y contempla la tasa de riesgo de pobreza después de transferencias sociales, la carencia material severa de bienes y los hogares que presentan muy baja intensidad laboral.

En un ámbito más global, ha tenido especial impacto el indicador de pobreza multidimensional del Oxford Poverty \& Human Development Initiative que dirige Sabine Alkire (https://ophi.org.uk/multidimensional-poverty-index/). El Índice examina más de 100 países y define la pobreza en función de cómo las personas se quedan atrás en tres dimensiones clave: salud, educación y nivel de vida, usando 10 indicadores como la falta de acceso a agua potable, nutrición adecuada o la educación primaria. Las personas que sufren privaciones en al menos un tercio de estos indicadores ponderados entran en la categoría de pobres multidimensionales.

El otro plano de la medición del desarrollo es la búsqueda de evidencias, una tendencia que se aprecia en todas sus disciplinas y que tiene que ver con la exigencia de rigor, con la dinámica de la investigación universitaria y sus incentivos y mecanismos para la divulgación y, también, especialmente en España, con las dificultades para que el desarrollo tengo el reconocimiento debido como disciplina en la evaluación de profesores, investigadores y universidades (Sianes 2016).

En la búsqueda de evidencias sobre el desarrollo es una novedad importante la fortaleza de la economía experimental y del comportamiento. La economía 
experimental estudia el comportamiento de las personas o los agentes económicas ante decisiones especificas o circunstancias o instrumentos de los mercados. Como señala Pablo Brañas, la economía ha estado estudiando el comportamiento de los individuos sin tener en cuenta estos pequeños detalles de la gente normal (Brañas 2011). La aplicación de la economía experimental al desarrollo ha tenido un notable auge e interesantes resultados que permiten medir el efecto de los comportamientos cooperativos, altruistas o egoístas, la aversión al riesgo o la propensión a diferir rentas en las intervenciones o programas de política pública o cooperación al desarrollo.

El impacto del premio Nobel de economía a Esther Duflo y Abhijit Banerjee ayuda a percibir el potencial de la economía experimental en algunos ámbitos del desarrollo y anima a medir con evidencias los resultados de las políticas. Duflo y Banerjee destacaban algunas conclusiones en su libro Repensar la pobreza que aportan perspectivas muy valiosas sobre los debates que hemos ido comentando. De sus evaluaciones concluyen que los países pobres no están destinados al fracaso porque sean pobres ni porque hayan tenido una historia desafortunada. Pero que es muy frecuente que los pobres carezcan de información fundamental y se crean cosas que no son ciertas, que se enfrenten cotidianamente a responsabilidad de demasiados aspectos de su vida o que las expectativas sobre lo que puede o no hacer la gente se conviertan a menudo en profecías autocumplidas (Banerjee y Duflo 2011). Aunque que sea difícil universalizar el resultado de los experimentos, en clave de políticas e intervenciones es de la máxima utilidad saber cuáles son los factores que tiene mayor incidencia en el comportamiento de las personas en entornos en desarrollo

\section{La emergencia de nuevos temas en las teorías del desarrollo}

Abarcar en un artículo el debate sobre la teoría y la agenda del desarrollo es una tarea imposible. Tampoco es fácil hacer una propuesta exhaustiva de los temas que parecen emergentes en la agenda del desarrollo a principios de la tercera década del SXXI. Cuatro de esos asuntos o problemas estarán sin duda presentes en las próximas décadas o ya reciben atención prioritaria: la desigualdad, el cambio climático, la transición tecnológica y energética y la política industrial.

El primero de ellos es la desigualdad, un problema clásico del desarrollo que recibe una vigencia mayor a la de cualquier otra época. La desigualdad siempre ha sido un problema central del desarrollo, pero su protagonismo tiene que ver con las 
diferencias entre el gráfico más popular en los años noventa sobre desigualdad que era la copa de Champagne del informe del año 2005 que representaba la distribución de renta mundial por quintiles, creando una gráfica con forma de copa y tallo quebradizo que ilustraba la mala distribución global de la renta por países.

La gráfica de esta década sobre desigualdad ha sido la curva del elefante de Branko Milanovic (2015) que representa la distribución por deciles del crecimiento del ingreso global en los años 1988-2008 y pone en evidencia la escasa rentabilidad de estos años de globalización expansiva para las clases medias de las economías más desarrolladas, en contraposición con la captura del crecimiento del ingreso por las clases medias de los países en desarrollo, reflejando particularmente la reducción de la pobreza y el crecimiento de las clases medias en China. La desigualdad, ese problema de los países en desarrollo, es ahora una variable principal en las economías occidentales.

Las publicaciones de gran impacto de Branko Milanovic, Thomas Piketty, Emmanuel Saez o Gabriel Zucman, se han unido a los conocidos trabajos de Angus Deaton o James Atkinson y otros autores, en un intenso debate al que se ha sumado Oxfam International con sus informes anuales emitidos en febrero para coincidir en las fechas en que se celebra el Foro Global Mundial de Davos.

Los datos son muy significativos. La desigualdad entre países se reduce, levemente si extraemos el caso chino, pero gana peso la desigualdad interna, sobre todo en las economías desarrolladas. Lo hace gracias, primero, a la deslocalización del empleo en el marco de la guerra por los costes y la eficiencia y después por el efecto de la precariedad de los mercados laborales y de las políticas de austeridad tras el estallido de la Gran Recesión. Asociado a la discusión sobre la precariedad y la exclusión, aparece la cuestión de la reforma impositiva y las políticas de redistribución, de gran vigencia en estos tiempos en los que la recuperación de la pandemia ha obligado a hacer un inédito esfuerzo fiscal. La precariedad y la exclusión se han combinado con el miedo al fin del trabajo por la automatización para abrir la reflexión sobre rentas básicas (y algunos experimentos). Una de las formulaciones que más preocupación genera en torno a estas cuestiones es la del ascensor social, un vehículo de progreso personal y social que parece perder fuerza conforme se exacerba la desigualdad y el estado tiene dificultades para redistribuir.

De los debates inspirados en la teoría, conviene destacar la falta de evidencias concluyentes sobre la curva de Kuznets que sostiene que hay un cierto grado de desigualdad deseable en las fases tempranas de crecimiento de una economía. No está claro y eso anima a discutir la relación entre crecimiento, pobreza y desigual- 
dad (Hill et al 2019). También se discute la medición de la desigualdad, dadas las limitaciones del índice de Gini. También los indicadores multidimensionales ganan peso en torno a la desigualdad, pues no sólo es de renta la desigualdad que limita el desarrollo de personas, comunidades o países. Las publicaciones de Piketty y los informes de Oxfam Internacional ponen en discusión si la desigualdad se debe medir en ingreso o en riqueza, poniendo en boga otra polémica muy activa como la imposición sobre el patrimonio y la sucesión.

El segundo de los asuntos emergentes es el del cambio climático que parece haber llegado ya a un punto de aceleración de algunos de sus problemas y que causa ya con cierta recurrencia algunas catástrofes naturales. Más allá de la necesidad de frenar inmediatamente algunas actividades cotidianas y productivas, ya sea mediante regulaciones o con incentivos, surgen las primeras evidencias de la vulnerabilidad de algunos países en desarrollo para mitigar y enfrentar los efectos de los desastres derivados del cambio climático.

El tercero de los asuntos emergentes es la transición ecológica y tecnológica que nace de la urgencia de actuar ante la evolución del cambio climático, pero está empujado también por la necesidad de las grandes potencias de competir globalmente y de encontrar sectores y actividades que sean motor de la recuperación post-pandemia. Es el fundamento del programa Next Generation UE y de los planes nacionales de recuperación y transformación de España y otros estados miembros de la UE. Tiene una concreción muy visible y factible en los países ricos, pero es más difícil prever si será viable en los países en desarrollo y en cuántos de ellos. El Pacto verde o Green Deal es la dimensión más visible pero la pandemia ha reforzado el compromiso por una triple transición (Sanahuja 2021) que incluye también una profunda apuesta por la transformación tecnológica y particularmente digital que parece ser uno de los ejes de futuro de la economía. Se incorpora finalmente la cohesión social como tercer elemento de la transición ante la incertidumbre ante los efectos de la automatización en el acceso al trabajo y ante las consecuencias combinadas de la gran recesión y la pandemia. Son tres ejes de modernización económica y social que tienes perfiles muy claros en los países desarrollados pero cuya viabilidad debe ser un debate importante en los próximos años. El acceso a las vacunas y las fórmulas de financiación de la deuda pública generadas por la crisis son sólo sus dos variables inmediatas.

Finalmente, el cuarto de los asuntos tiene que ver la necesidad de una nueva política industrial. Según Rodrik, los países en desarrollo experimentan una prematura desindustrialización y se terciarizan antes de impulsar la industria (Rodrik y Stantcheva 2022). Los países, en estas últimas décadas en las que las políticas 
sectoriales perdieron peso frente a las instrumentales, han abordado políticas de reducción de la pobreza invirtiendo en educación y formación (pre-producción) y en redes de seguridad social o políticas de promoción de empleo y transferencias de renta ((post-producción). Después han desarrollo políticas enfocadas en la industrialización y el crecimiento, promoviendo acuerdo de libre comercio o sistemas de innovación (pre-producción), o subsidios e incentivos a para I+D (en producción) o incentivos fiscales (post-producción).

Rodrik propone con Stantcheva y otros coautores un nuevo tipo de políticas industriales para trabajar en la dimensión intermedia de las políticas de fomento de la productividad, (Good Jobs Development Model), escapando de la concentración en los sectores de baja productividad que no generan crecimiento sostenible y de los sectores de alta productividad que no son capaces de arrastrar al resto de la economía al crecimiento.

Se trataría de un modelo basado en medidas de apoyo y fomento en la fase de producción orientados a crear empleo de mayor productividad a partir de una relación colaborativa e iterativa con las empresas a la que se facilite asistencia personalizada con condiciones suaves de creación de empleo, no especialmente centrado en las pequeñas y medianas empresas, sino en las empresas de servicios.

La política industrial es un tema recurrente en la literatura reciente sobre desarrollo. Aunque la propuesta de Estado Emprendedor de Mariana Mazzucato no se centra en la promoción de sectores productivos sino en la promoción de ecosistemas de innovación público-privados, apuesta por un Estado que adopte un rol activo en la promoción de la innovación, en lugar de limitarse a reducir el riesgo para el sector privado y corregir los fallos del mercado (Mazzucato 2019). Igual que Stiglitz - Justin Lin, Ha-Joon Chang se une también a la reclamación de la vuelta de la política industrial, que se había convertido, afirma, en una frase vacía y vuelve a estar de actualidad tanto en el ámbito académico como en la política. Su propuesta busca huir de los condicionantes ideológicos que dominaban el debate en las últimas décadas y quiere optimizarla respuesta a nuevos desafíos que exceden en ocasiones la capacidad de los estados como, señalan, las guerras comerciales, el tamaño de los gigantes económicos tipo Amazon o Apple o la desinversión que genera la financiarización de las grandes empresas industriales, que están en ocasiones más atentas a las operaciones bursátiles que a la producción (Chang y Andrenoni 2020). 


\section{Conclusiones}

Hemos afirmado que la evolución del desarrollo en estos setenta y cinco años ha sido positiva por dos razones. La primera es que pese a la percepción fatalista que produce la cercanía a la información y a los problemas, las variables del desarrollo tienden a mejorar persistentemente y hay cada vez menos gente en situación de pobreza. Es cierto que en torno a mil millones de personas están en situación de pobreza extrema o pobreza severa y esa cifra es excesiva por mucho que hay decrecido. La gravedad de algunos problemas y conflictos infringe un sufrimiento intolerable a muchas personas en el mundo. La evolución del cumplimiento de los ODS provoca sensación de impaciencia y una cierta inseguridad sobre si avanzamos lo suficiente. La pandemia, como hizo la Gran Recesión, ha demostrado la debilidad de muchos de los avances que celebrábamos, comprometiendo de nuevo el futuro de mucha gente. El mundo mejora, pero los riesgos de involución son significativos y muy costosos para los más vulnerables.

La segunda razón para sustentar un balance positivo es que la sociedad mundial fue capaz de dotarse de un consenso global multidimensional, pertinente y comprometido con el objetivo de no dejar a nadie atrás. Antes de los años 90, el panorama del desarrollo estaba marcado por dilemas basados en un cierto dogmatismo que perjudicaba la eficacia de las políticas. El consenso global, representando hoy en los ODS, es por eso una buena noticia, pese a que su ambiciosa formulación nos haga dudar de su cumplimiento. La evolución de la Gran Recesión y de la pandemia podría dar lugar a un cambio de paradigmas y escenarios más favorable para el consenso global, si recuperamos la fortaleza del sistema multilateral de relaciones internacionales y si los asuntos emergentes de la agenda del desarrollo evolucionan positivamente.

Los retos de futuro están asociados al cumplimiento de los objetivos de la agenda 2030 que dependerá mucho de la eficacia de unas políticas que se deben apoyar en la pertinencia de los instrumentos teóricos aplicados a cada problema en cada entorno específicos, así como de la capacidad de evaluar con evidencias solidas sus diseños y resultados. El margen de mejora del conocimiento sobre el desarrollo y sus políticas es todavía amplio, igual que el de los mecanismos de cooperación internacional $y$ ese es un reto de futuro.

Otro reto, que no hemos abordado, pero se va a manifestar seguro con creciente fuerza, es el de la descolonización del pensamiento sobre desarrollo. Las teorías, los expertos, las publicaciones sobre desarrollo tienen un claro sesgo hacia las percepciones, modelos y personas de los países más desarrolladas. 
Las voces de los países en desarrollo tienen todavía menos presencia e influencia de lo debido y ese sesgo deberá ser corregido (Ndlovu-Gatsheni 2018). Quizás el nuevo consenso global tras la agenda 2030 tendrá que preguntarse cómo atender un orden global marcadamente cambiado en el siglo XXI o si hay que buscar alternativas más pragmáticas para los deseos de equidad y bienestar de la gente y desligarlos del modo occidental de vivir. Podría ser necesario otorgarle mayor centralidad al Buen Vivir/Sumak Kawsay/Suma Qamaña de América Latina, o el Swaraj de la India.

También en los países occidentales podemos encontrar argumentos en favor de otras visiones del desarrollo, que rechacen la modernidad capitalista como se ha querido ver en algunas respuestas a la restricción de movimientos en la pandemia. Cabe, por tanto, preguntarse si la formulación del próximo consenso, que podría definir un alcance de 2030 a 2045, no deberá ser más rupturista y alternativo que el que hoy nos hemos dado

\section{Bibliografía}

Acemoglu, D. y Robinson A. (2021). ¿ Por qué fracasan los países?, Deusto.

BANerJeE, A. y DufLO, E. (2011). Repensar la pobreza: un giro radical en la lucha contra la desigualdad global, Taurus.

Birdsall, N., de la Torre, A. y Valencia, F. (2010). The Washington Consensus: Assessing a Damaged Brand. CGD Working Paper 213. Center for Global Development. https://www.cgdev.org/publication/ washington-consensus-assessing-damaged-brand-working-paper-213

Brañas, P. (201 1). Economía Experimental y del Comportamiento, Antoni Bosch.

Chang, H.-J., Andreoni, A. (2020). "Industrial policy in the 21 st Century", Development and Change. Volume 51, Issue2, FORUM 2020, marzo, 324-351.

CONSEJO DE REDACCIÓN RFS (2005). "Desarrollo económico y social: teorías, propuestas, responsabilidades", Revista de Fomento Social 60, 11-38.

CONSEJO DE REDACCIÓN RFS (2010). "Ser hermanos más allá de la globalización. El desarrollo humano integral en Caritas in Veritate", Revista de Fomento Social 65, 189-204. 
Currie-Alder, B., Kanbur, R., Malone, D. M. and Medhora, R. (2014). International Development: Ideas, Experience, and Prospects, Oxford University Press.

Harris, J. (2014). "Development Theories", en Currie-Alder, B., Kanbur, R., Malone, D. M. and MEDHORA, R. (2014) International Development: Ideas, Experience, and Prospects, 35-49, Oxford University Press.

Hidalgo-CAPITÁn, A. L. (1998). El pensamiento económico sobre desarrollo. De los mercantilistas al PNUD, Servicio de Publicaciones de la Universidad de Huelva, Huelva.

Hills, J., McKnight, A. Bucell, I., Karagiannaki, E., Vizard, P., Yang , L. with Duque, M. and RUCCI, M (2019). "Understanding the relationship between poverty, inequality and growth: Case report 119", Overview Report, London School of Economics, London.

JIMÉNEZ, J. C. (2013). "Etapas del desarrollo de la economía mundial", capítulo 2 en Alonso, J. A. (dir.), Lecciones sobre Economía Mundial. Introducción al desarrollo y a las relaciones económicas e internacionales, 59-86, Madrid, Thomson Reuters.

KLIEMANN, C, (2018). "Why We Need Alternatives to Development". Blog EADI, 22 de noviembre, http://wordpress.p288574. webspaceconfig.de/?p=239\#more-239

LeVITSKY, S. y Ziblatr, D. (2018). Cómo mueren las democracias, Ariel.

MazzucAto, M. (2019). El estado emprendedor: mitos del sector público frente al privado, RBA.

MILANOVIC, B. (2011). Los que tienen y los que no tienen. Una breve y singular historia de la desigualdad global, Alianza.

MILANOVIC (2012). Global Income Inequality by the Numbers: In History and Now, an overview, Policiy Research Working Paper WPS6259, Worldbank, http://documents.worldbank.org/curated/en/959251468176687085/ Global-income-inequality-by-the-numbers-in-history-and-now-an-overview

Ndovu-GatshenI, S. (2018). "The dynamics of epistemological decolonisation in the 21 st century: towards epistemic freedom", Strategic Review for Southern Africa, vol. $14, n^{\circ} 1$. 
Payne. A. y Phillips N. (2010). Desarrollo. Alianza.

PINKER, S. (2018). En defensa de la ilustración. Paidós.

PNUD (1992). Informe sobre Desarrollo Humano 2010. Tercer Mundo, Colombia.

- (2010). Informe sobre Desarrollo Humano 2010. La verdadera riqueza de las naciones: Caminos al desarrollo humano, Mundi-Prensa.

- (2020). Informe sobre Desarrollo Humano 2020. La próxima frontera. El antropoceno y el desarrollo humano, Programa de Naciones Unidas para el desarrollo.

RODRIK, D. (2015). Economics Rules: The Rights and Wrongs of The Dismal Science, New York, W.W. Norton.

RODRIK D., and BLANCHARD, O. ed. (2021). Combating Inequality: Rethinking Government's Role. Cambridge, MA, MIT Press.

RodRIK, D. y StantCheVA, S. (2022). "Fixing Capitalism's Good Jobs Problem", Oxford Review of Economic Policy, forthcoming.

SanAHUja, J. A. (2021). "Pacto Verde Europeo: el giro ambiental de un actor global", en CEIPAZ (2021). El mundo después de la pandemia: enfrentar la desigualdad y proteger el planeta. Anuario CEIPAZ 2020-2021, 69-96, Madrid, Ceipaz.

Secretariado para la justicia social y la ecología. Grupo de trabajo sobre economía (2016). "Informe especial: Por una economía global justa. Construir sociedades Sostenibles e inclusivas", Promotio iustitiae 121, 2016/1.

SIANES, A. (2016). "Estudios sobre la creación de un área de conocimiento en estudios para el desarrollo", Informe para Red española de estudios para el desarrollo REEDES, junio.

Stewart, F. and Samman, E. (2015). "Inequality and Development: an Overview", en CURRIE-Alder, Kanbur, Malone y Medhora, International Development: Ideas, experiencies and prospects, Oxford University Press.

Stigutz, J. E., Sen, A. and Fitoussi, J. P. (2009). Report by the Commission on the Measurement of Economic Performance and Social Progress, en https://www. insee.fr/en/information/2662494 [acceso julio 2017]. 
Tezanos, S., QuiÑones, A., GutiéRrez, D. y MAdRUeÑo, R. (2013). Desarrollo humano, pobreza y desigualdades. Manuales sobre cooperación y desarrollo, Cátedra de Cooperación Internacional y con Iberoamérica, Universidad de Cantabria.

TODARO, M. (1988). El desarrollo económico del Tercer Mundo, Alianza.

WiLIAMsON, J. (1990). What Washington Means by Policy Reform, Latin American adjustment: how much has happened? ('Washington D. C.: Instituto de Economía Internacional.

- (2004). "A Short History of the Washington Consensus". Paper commissioned by Fundación CIDOB for a conference "From the Washington Consensus towards a new Global Governance", Barcelona, September, 24-25.

YOH, S. (2019). "5 Things that will Frustrate the Heck out of you when studying International Development", Blog EADI, 17 de julio, http://www.developmentresearch.eu/? $\mathrm{p}=469$

Yusuf, S. (2014). "Fifty Years of Growth Economics", en CuRRIE-Alder, B., KanBur, R., MALONE, D. M. and MeDHORA, R. (2014) International Development: Ideas, Experience, and Prospects, 50-64, Oxford University Press. 